\title{
ASPECTOS EPIDEMIOLÓGICOS DA TUBERCULOSE PULMONAR NOS MUNICIPIOS DE BOTUCATU, CONCHAS, SÃO MANUEL E AVARÉ, ESTADO DE SÃO PAULO, BRASIL, DE 1963 A 1972 *
}

\author{
Cecilia Magaldi** \\ Ivete Dalben Soares *** \\ Sheila Zambello de Pinho ****
}

RSPU-B/325

MAgaldi, C. et al. - Aspectos epidemiológicos da tuberculose pulmonar nos municipios de Botucatu, Conchas, São Manuel e Avaré, Estado de São Paulo, Brasil, de 1963 a 1972. Rev. Saúde públ., S. Paulo, 10:291-314, 1976.

Resumo: O diagnóstico da situação da tuberculose pulmonar nos Municipios de Botucatu, Conchas, São Manuel e Avaré, SP, Brasil, baseou-se na prevaléncia $e$ risco de infecção em escolares de $1^{\text {* }}$ série, em 1972, e em dados de morbidade e mortalidade especifica, de 1963 a 1972. Realizou-se inquérito tuberculinico em 2.913 escolares, com PPD 23 RT-2UT; foram analisados os prontuários de 718 casos inscritos no CSI de Botucatu e os atestados de óbito de residentes, incluindo-se os ocorridos em hospitais especializados. As taxas de prevalência de infecção para os quatro Municipios foram respectivamente $2,4 \% ; 6,8 \% ; 1,9 \%$ e 4,5\%. Para a idade de 7,5 anos, os riscos de infecção foram: $0,27 \% ; 0,32 ; 0,20 \%$ e $0,34 \%$. O nivel de infecção apurado caracterizon o conjunto como área de média prevalencia da tuberculose pulmonar. Os indices de prevalência de infecção relacionaram-se diretamente à incidência de casos baciliferos de cada Municipio. Em 530 casos com baciloscopia, houve 62,0\% de positividade. As formas radiológicas moderada e avançada predominaram principalmente em adultos jovens do sexo masculino. Foram registradas alta taxa de abandono (39,5\%), baixo percentual de cura $(17,9 \%)$ e 3,4\% de óbitos; ao redor de 32,0\% estava em tratamento. Não houve diferenças estatisticamente significativas das proporções de abandono, segundo procedência por Município ou formas da doença. Invocaram-se razóes técnico-administrativas do sistema vigente para explicar esses achados. A incidência média de casos confirmados, no periodo, foi de 35,4 em Conchas, 33,1 em Avaré, 23,7 em Botucatu e 18,5 em São Manuel (por 100.000). Com casos confirmados e suspeitos os indices foram: 57,$6 ; 48,8 ; 43,8$ e 35,3. Os indices médio-anuais de mortalidade, foram: $7,6 \mathrm{em}$ Botucatu, 12,14 em Conchas, 5,0 em São Manuel e 18,7 em Avaré (por 100.000). Apenas em Botucatu registrou-se declinio das taxas de mortalidade de 1963 a 1972. A mortalidade especifica em adultos jovens ainda era elevada, predominando no sexo masculino, principalmente em Conchas. Sublinhou-se a necessidade de descentralizar as atividades antituberculose para atingir um controle eficiente.

UNITERMos: Tuberculose pulmonar. Inquérito tuberculinico, escolares. Morbidade e mortalidade. Epidemiologia.

* Trabalho baseado na Tese de Livre-Docência apresentada à Faculdade de Ciências Médicas e Blológicas de Botucatu por Cecilia Magaldi, realizado com auxílio da Funđação de Amparo à Pesquisa do Estado de São Paulo, processos n.os 72/227 e 73/117.

** Do Departamento de Medicina em Saúde Pública da Faculdade de Ciências Médicas e Biológicas de Botucatu da UNESP - Rubião Júnior - Botucatu, SP - Brasil.

* * Acadêmica de Medicina da Faculdade de Ciências Médicas e Biológicas de Botucatu.

**** Do Departamento de Matemática da Faculdade de Ciências Médicas e Biologicas de Botucatu. 
MAGALDI, C. et al. - Aspectos epidemiológicos da tuberculose pulmonar nos municípios de Botucatu, Conchas, São Manuel e Avaré. Estado de São Paulo, Brasi], de 1963 a 1972. Rev. Saúde públ., S. Paulo, 10:291-314. 1976.

\section{INTRODUCĀO}

A política de integração das atividades anti-tuberculose aos serviços gerais de saúde confinou-se a áreas muito restritas do Brasil "s.

Ao mesmo tempo faltou, até recentemente, uma "organização sistêmica econômica e administrativa suficiente e capaz" ${ }^{39}$ para assumir o controle da tuberculose pulmonar.

Como consequiência desses fatos e também do progressivo deslocamento da tuherculose do meio urbano para o rural, onde ela é controlada ainda menos i, 39 , os índices de morbidade e mortalidade. embora tenham declinado nos últimos 30 anos, configuram uma situação ainda desfavorável para o Brasil. em relação a outros países ${ }^{39}$.

Os conhecimentos sobre a epidemiologia da tuberculose pulmonar, no interior do Estado de São Paulo, são ainda escassos a julgar pelo pequeno número de publicaçoes a respeito $\bar{\nu}, 6,-, 9,23,36,41,-\cdots$.

A fase atual da transição do sistema vertical para o de integração das atividades de controle da tuherculose, no Estado de São Paulo ${ }^{50}$. aliada à carência de informes epidemiológicos justificaram. assim, uma investigação para se definir a situação da tuherculose em uma área do interior do Estado.

As informaçóes colhidas para o período escolhido podem ser úteis no futuro como termo de comparação para estudos do efeito. a médio e longo prazo, do sistema de controle em implantação.

Os objetivos deste trabalho, visando a um diagnóstico da situação da tuberculose pulmonar nos municípios de Botucatu, Conchas, São Manuel e Avaré, foram os seguintes:

1. definir. por meio de inquérito tuberculinico. o nível de infecção tuberculosa em escolares de 1 . $^{\text {a }}$ série, em 1972. e estimar o risco anual de inferção:
2. conhecer a morbidade por tuberculose pulmonar nos quatro Municípios, no período de 1963 a 1972, com base em dados de fichas e prontuários médicos de pacientes matriculados na área de Tisiologia do Centro de Saúde I (CSI) de Botucatu;

3. mediante análise de atestados de óbito. conhecer a mortalidade específica por tuberculose pulmonar, no mesmo período.

\section{MATERIAL E MÉTODOS}

Definição da área

A Divisão Regional de Saúde de Sorocaba (DRS-4) compreende sete Distritos Sanitários, entre os quais o de Botucatu (DS-7) e o de Avaré (DS-6), cada um com oito municípios ${ }^{56,58}$.

Foram selecionados, como áreas de referência para este estudo, o município de Araré (DS-6) e os de Botucatu, Conchas e São Manuel, pertencentes ao DS-7, em rista do maior contingente de escolares e da rariação das distâncias de Conchas, São Manuel e Avaré com relação à Botucatu i distâncias respectivas por rodovia: 54.25 e $75 \mathrm{~km}$ ).

As atividades de controle de tuberculose eram exercidas, exclusivamente, pelo CSI de Botucatu.

\section{Inquérito tuberculínico}

Fase de preparação (de 29/02/1972 a $0704 / 1972$ ) - Foi feita aferição, por leitora-padrão, de uma atendente já treinada na técnica de aplicação e leitura da prova tuberculinica segundo as normas da Divisão Nacional de Tuberculose (DNT). em 1970. Além disso, foram realizadas visitas às escolas de cada municipio. 78 palestras rápidas a grupos de professores e diretores, 11 palestras em 
MAGALDI, C. et al. - Aspertos epidemiológicos da tuberculose pulmonar nos municípios de Botucatu, Conchas, São Manuel e Avaré, Liuduo de Sao Paulo, Brasill, de 1963 a 1972. Rev. Saúde públ., S. Paulo, 10:291-314, 1976.

reuniões de pais e mestres e 4 entrevistas em emissoras de rádio. Foram distribuídas cartas solicitando autorização de pais ou responsáveis, para aplicação do teste tuberculínico.

Fase de execução $(10 / 04 / 1972$ a $23 / 07 / 1972$ ) - Submeteram-se ao teste, escolares de todas as idades matriculados na $1 .^{a}$ série de 12 escolas urbanas públicas e privadas de Botucatu, 2 de Conchas, 5 de São Manuel e 7 de Avaré. Nas Tabelas 1 e 2 encontram-se a distribuição da população matriculada, segundo a cidade e o grupo etário, assim como os percentuais de cobertura e rendimento atingidos em cada idade e para o total das idades.

A prova tuberculinica foi efetuada de acordo com a 2." Recomendação da Comissão Técnica da Campanha Nacional contra a Tuberculose ${ }^{14}$, utilizando-se como antígeno o PPD 23RT Tween 80, 2 UT $(0,04 \mathrm{mcg}$ por $0,01 \mathrm{ml})$. Foram utilizadas seringas "Omega Microstat" e agulhas "Unicef Luer Mount" calibre 26 e $13 \mathrm{~mm}$ de comprimento.

Consideraram-se como positivas as reações com induração de 10 ou mais $\mathrm{mm}$ (reator-forte), como fracas as de 5 a 9 $\mathrm{mm}$ (reator-fraco) e como negativas as de 0 a $4 \mathrm{~mm}$, à leitura de 72 horas.

0 índice de prevalência de infecção, em percentagem, foi expresso como a razão entre o número de reatores-fortes $e$ o número de testes aplicados e lidos, em cada grupo etário, para cada cidade ${ }^{16,44}$.

Os índices de incidência de infecção por idade (risco anual de infecção) foram obtidos por meio de duas fórmulas:

$$
\begin{aligned}
& \text { 1) } i=\frac{P}{t} \\
& \text { 2) } P(t)=1-(1-i)^{t}
\end{aligned}
$$

onde $\mathrm{P}=$ prevalência, $\mathrm{i}=$ incidência, $\mathrm{t}=$ idade média do grupo etário examinado.

Utilizou-se a máquina "Hewlett-Packard 9.100B" do Centro de Processamento de Dados da Faculdade de Ciências Médicas e Biológicas de Botucatu (FCMBB), para a realização dos cálculos da fórmula 2 .

De 19 a 22 de março de 1973 foi repetido o inquérito tuberculínico na mesma população escolar examinada em 1972. nos municípios de Conchas e São Manuel. A aplicação e leitura da prova ficaram a cargo de duas leitoras-padrão do Departamento de Assistência Escolar da Prefeitura Municipal de São Paulo, cedidas pela Disciplina de Tisiologia da Faculdade de Saúde Pública da USP. Para os dois municipios, a cobertura em 1973 foi de $88,0 \%$ e o rendimento de $95,4 \%$.

\section{Morbidade}

A casuística consistiu em 718 casos matriculados pela primeira vez com diagnóstico de tubercuolse pulmonar (suspeita ou comprovada) no CSI de Botucatu, no período de $1 .^{\circ}$ de janeiro de 1963 a 31 de dezembro de 1972, todos residentes em Botucatu, Conchas, São Manuel e Avaré.

As informações relativas aos 718 casos foram colhidas dos livros de registro mensal de casos novos, fichas de visitação domiciliária, prontuários médicos e fichas Mc Bee-Keysort modelo único do serviço de abreugrafia.

A colheita de dados estendeu-se de $10^{\circ}$ de julho a 30 de outubro de 1973, tendo sido os mesmos codificados e passados para cartōes IBM, para posterior processamento em computador IBM $113016 \mathrm{~K}$, da Unidade de Processamento de Dados da Escola Superior de Agricultura Luiz de Queiroz (USP).

A classificação das formas radiológicas de tuberculose empregada no CSI de Botucatu baseou-se nos padrôes diagnósticos 
MAGALDI, C. et al. - Aspectos epidemiológicos da tuberculose pulmonar nos municipios de Botucatu, Conchas, São Manuel e Avaré, Estado de São Paulo, Brasil, de 1963 a 1972. Rev. Saúde públ., S. Paulo, 10:291-314, 1976.

\section{TA B E L A 1}

Inquérito tuberculínico em escolares de 1. série primária de escolas urbanas públicas e particulares, 1972 - Cobertura da aplicação da prova tuberculínica por cidade e por grupo etário

\begin{tabular}{|c|c|c|c|c|c|c|c|}
\hline \multirow{8}{*}{ 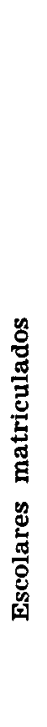 } & & lade & Botucatu & Conchas & S. Manuel & Avaré & Total \\
\hline & 6 & $\begin{array}{l}N .0 \\
\%\end{array}$ & $\begin{array}{l}475 \\
30,1\end{array}$ & $\begin{array}{l}44 \\
20,5\end{array}$ & $\begin{array}{l}98 \\
20,0\end{array}$ & $\begin{array}{l}61 \\
6,4\end{array}$ & $\begin{array}{c}678 \\
20,9\end{array}$ \\
\hline & 7 & $\begin{array}{l}\text { N.o } \\
\%\end{array}$ & $\begin{array}{l}656 \\
41,6\end{array}$ & $\begin{array}{l}89 \\
41,4\end{array}$ & $\begin{array}{l}199 \\
40,5\end{array}$ & $\begin{array}{l}\mathbf{5 1 6} \\
\mathbf{5 4 , 4}\end{array}$ & $\begin{array}{r}1.460 \\
45,1\end{array}$ \\
\hline & 8 & $\begin{array}{l}\text { N.o } \\
\%\end{array}$ & $\begin{array}{l}228 \\
14,4\end{array}$ & $\begin{array}{l}51 \\
23,8\end{array}$ & $\begin{array}{l}93 \\
18,9\end{array}$ & $\begin{array}{l}194 \\
20,4\end{array}$ & $\begin{array}{c}566 \\
17,5\end{array}$ \\
\hline & 9 & $\begin{array}{l}\text { N.o } \\
\%\end{array}$ & $\begin{array}{r}104 \\
6,6\end{array}$ & $\stackrel{15}{7,0}$ & $\begin{array}{l}49 \\
10,0\end{array}$ & $\begin{array}{l}89 \\
9,3\end{array}$ & $\begin{array}{r}257 \\
7,9\end{array}$ \\
\hline & $10+$ & $\begin{array}{l}\text { N.o } \\
\%\end{array}$ & $\begin{array}{r}108 \\
6,8\end{array}$ & $\begin{array}{l}16 \\
7,5\end{array}$ & $\begin{array}{l}53 \\
10,8\end{array}$ & $\begin{array}{l}87 \\
9,1\end{array}$ & $\begin{array}{r}264 \\
8,1\end{array}$ \\
\hline & NR & $\begin{array}{l}\text { N.o } \\
\%\end{array}$ & $\begin{array}{l}7 \\
0,4\end{array}$ & - & - & - & - \\
\hline & $\begin{array}{c}\text { Total } \\
(100 \%)\end{array}$ & N.o & 1.578 & 215 & 492 & 947 & 3.232 \\
\hline \multirow{7}{*}{ 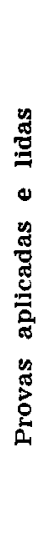 } & 6 & $\begin{array}{l}\text { N.O } \\
\%\end{array}$ & $\begin{array}{l}430 \\
30,5\end{array}$ & $\begin{array}{l}39 \\
20,5\end{array}$ & $\begin{array}{l}92 \\
20,2\end{array}$ & $\begin{array}{c}56 \\
6,5\end{array}$ & $\begin{array}{c}617 \\
21,1\end{array}$ \\
\hline & 7 & $\begin{array}{l}\mathbf{N .} \\
\%\end{array}$ & $\begin{array}{l}603 \\
42,7\end{array}$ & $\begin{array}{l}82 \\
43,0\end{array}$ & $\begin{array}{l}188 \\
41,1\end{array}$ & $\begin{array}{c}\mathbf{4 7 5} \\
\mathbf{5 5 , 6}\end{array}$ & $\begin{array}{r}1.348 \\
46,2\end{array}$ \\
\hline & 8 & $\begin{array}{l}\text { N.o } \\
\%\end{array}$ & $\begin{array}{l}197 \\
14,0\end{array}$ & $\begin{array}{l}42 \\
22,0\end{array}$ & $\begin{array}{l}85 \\
18,6\end{array}$ & $\begin{array}{l}169 \\
19,8\end{array}$ & $\begin{array}{c}493 \\
16,9\end{array}$ \\
\hline & 9 & $\begin{array}{c}\mathrm{N} .{ }^{\circ} \\
\%\end{array}$ & $\begin{array}{l}89 \\
6,3\end{array}$ & $\begin{array}{c}14 \\
7,4\end{array}$ & $\begin{array}{l}46 \\
10,1\end{array}$ & $\begin{array}{l}77 \\
9,0\end{array}$ & $\begin{array}{r}226 \\
7,7\end{array}$ \\
\hline & $10+$ & $\begin{array}{c}\text { N.o } \\
\%\end{array}$ & $\begin{array}{l}89 \\
6,3\end{array}$ & $\begin{array}{c}14 \\
7,4\end{array}$ & $\begin{array}{l}46 \\
10,1\end{array}$ & $\begin{array}{l}76 \\
8,9\end{array}$ & $\begin{array}{r}225 \\
7,7\end{array}$ \\
\hline & NR & $\underset{\%}{N}{ }^{\circ}$ & $\begin{array}{l}4 \\
0,3\end{array}$ & - & - & - & 二 \\
\hline & $\begin{array}{l}\text { Total } \\
(100 \%)\end{array}$ & N.o & 1.412 & 191 & 457 & 853 & 2.913 \\
\hline \multirow{7}{*}{$\begin{array}{l}20 \\
8 \\
0 \\
0 \\
0 \\
0 \\
0 \\
0\end{array}$} & 6 & & 90,5 & 88,7 & 93,9 & 91,8 & 91,0 \\
\hline & 7 & & 90,5 & 92,2 & 94,5 & 92,0 & 92,3 \\
\hline & 8 & & 85,9 & 82,4 & 91,4 & 87,1 & 87,1 \\
\hline & 9 & & 85,5 & 93,4 & 93,9 & 86,5 & 87,9 \\
\hline & $10+$ & & 88,1 & 87,5 & 86,8 & 87,3 & 85,6 \\
\hline & NR & & 57,1 & 一 & $\rightarrow$ & - & 一 \\
\hline & Total & & 89,5 & 88,9 & 92,9 & 90,0 & 90,1 \\
\hline
\end{tabular}

NR = Idade não referida. 
MAGALDI, C. et al. - Aspectos epidemiológicos da tuberculose pulmonar nos municipios de Botucatu, Conchas, São Manuel e Avaré, Estado de São Paulo, Brasil, de 1963 a 1972. Rev. Saúde pübl., S. Paulo, 10:291-314, 1976.

T A B ELA 2

Inquérito tuberculínico em escolares de 1 a $^{\text {a }}$ sie primária de escolas urbanas públicas e particulares, 1972 - Rendimento da aplicação da prova tuberculinica por cidade e por grupo etário

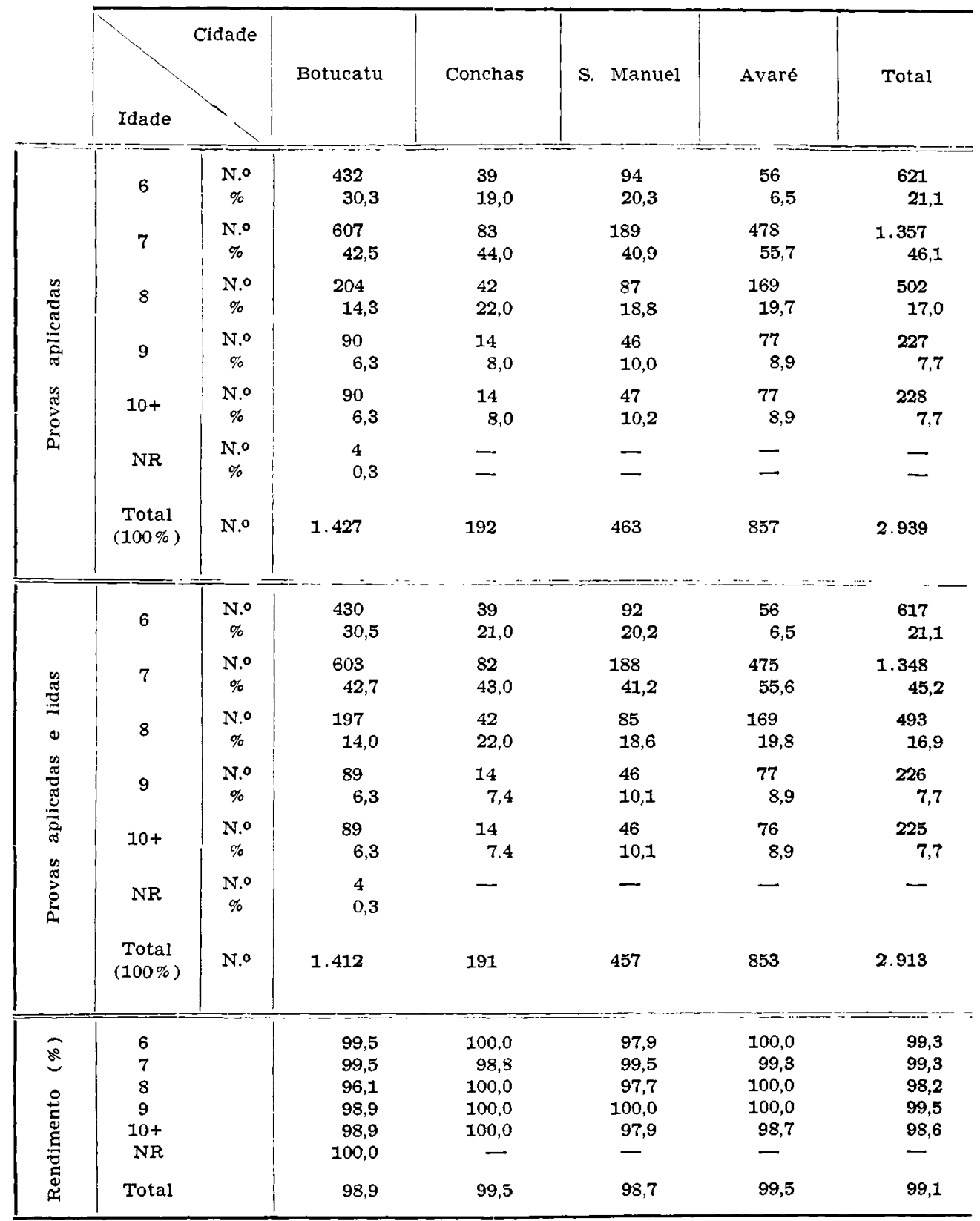


MAGALDI, C. et al. - Aspectos epidemiológicos da tuberculose pulmonar nos municípios de Botucatu, Conchas, São Manuel e Avaré, Estado de São Paulo, Brasil, de 1963 a 1972. Rev. Saúde publ., S. Paulo, 10:291-314, 1976.

da "National Tuberculosis Association" i1.

O diagnóstico de "tuberculose primária" feito de rotina dependia de uma induração $\geqslant 10 \mathrm{~mm}$ à prova de Mantoux e da presença ou não de lesão radiológica respectivamente tuberculose primária "com sombras" e "sem sombras".

Os tipos de evolução estabelecidos foram: cura, recaída, abandono, óbito, transferência para internação, transferência para outros serviços e em tratamento. Não existia a designação de "crônico" nas fontes de informação utilizadas e para 31 casos não havia informação sobre a evolução. Eram obedecidos os critérios vigentes para definição de $\operatorname{cura}^{59,02,64}$.

Foram computados como casos "em tratamento" todos os pacientes que assim estavam classificados no período da colheita de dados e incluíam aqueles que: 1) recebiam medicação específica dentro ou fora dos prazos preconizados pelas normas vigentes ${ }^{59,61,62,04} ; 2$ ) estavam em controle pós-tratamento, e 3) tinham abandonado o tratamento ou o controle, mas o abandono ainda não havia sido registrado pelo serviço.

O abandono era considerado como sendo o não comparecimento do paciente decorridos 6 meses da última consulta na fase de tratamento e 12 meses na fase de rontrole pós-tratamento.

Com relação aos regimes de tratamento adotava-se o difásico ${ }^{57,59,63,64}$ oul o amhulatorial desde o início (monofásico), dependendo de condições de jsolamento domiciliar. aceitação de internação ou da existência de vagas em hospitais.

Foram estimados dois tipos de indices de incidencia: 1) o índice obtido da razão entre o número de "casos de tuberculose". segundo os critérios da OMS ${ }^{40}$ * da Comissão Técnica da Campanha Nacional contra a Tuberculose ${ }^{15}$, isto é, com haciloscopia positiva e a população correspondente segundo idade e sexo; 2) o indice II. ohtido da razão entre o número total de casos novos tanto bacilíferos como aqueles sem confirmação bacterioló- gica (casos suspeitos) e a população correspondente. Os resultados foram expressos por 100.000 habitantes. Para efeito dessas estimativas foram incluídos somente os casos novos cujas lesões foram diagnosticadas como: "tuberculose primária com sombras", lesão mínima, moderada, avançada e derrame pleural, num total de 557 casos, excluindo-se 152 casos de "forma primária sem sombras" e 9 casos de forma não especificada.

A estimativa das populações por grupo etário e sexo, de 1963 a 1972, baseou-se nos dados dos censos de 1960 e 1970, sendo necessário antes recorrer à média aritmética das populações totais e por idade e sexo, de 1950 e 1970, visto não haver dados de população masculina e feminina para 1960.

Com as médias aritméticas foi feito o pró-rateio das diferenças em relação às populações, por idade, do censo de 1960.

Utilizou-se o método geométrico ${ }^{11} \mathrm{pa}$ ra a estimativa das populações de 1963 a 1972.

Para o tratamento estatístico no estudo de associação de variáveis: municípios, formas de tuberculose e abandono de tratamento foi empregado o teste do qui-quadrado com $\alpha=0.05^{\circ 0}$.

\section{Mortalidade especifica}

As informações sobre óbitos por tuberculose pulmonar segundo local de residência, ano de ocorrência, idade e sexo para Botucatu. Conchas e São Manuel foram colhidas dos mapas demógrafo-sanitários, e para Avaré. diretamente dos atestados de óbito originais. no Cartório de Registro Civil.

Foi obtida. dos 9 hospitais de Tisiologia do Estado de São Paulo, a relação dos óbitos por tuberculose pulmonar ocorridos nesses hospitais segundo municipio, ano. idade e sexo.

Para a estimativa dos índices de mortalidade foram utilizadas as mesmas po- 
MAGALDI, C. et al. - Aspectos epidemiológicos da tuberculose pulmonar nos municipios de Botucatu. Conchas, São Manuel e Avaré. Estado de São Paulo, Brasil, de 1963 a 1972. Rev. Saúde pubt. S. Paulo, 10:291-31t. 1976.

pulações totais e por idade e sexo. sendo o numerador a soma dos óbitos que constavam nos mapas ou atestados e mais os ocorridos nos hospitais.

\section{RESULTADOS E DISCLSSAOO}

A Tabela 3 mostra que a maior prevalência de infecçũo para todas as idades foi encontrada em Conchas $(6.81 \%)$. seguindo-se Avaré $(4.57 \%)$. Botucatu $(2.41 \%)$ e São Manuel $(1.97 \%)$, estimando-se em $3.26 \%$ a prevalência para os quatro municípios.

Comparativamente aos resultados obtidos em escolares de $1 .^{\mathrm{a}}$ série primária, nas capitais brasileiras. em $1971^{3}$. verifica-se que os índices de Botucatu. São Manuel e Avaré foram inferiores aos de Curitiba $\left(6.4^{\circ} \mathrm{C}\right)$ e São Paulo $(6.6 \%)$, os menores dentre as capitais. enquanto o de Conchas foi algo superior. Os índices de Conchas e Araré foram semelhantes aos dados disponiveis para outras cidades do interior do Estado de São Paulo em épocas próximas ${ }^{5,6,}$,

Diferenças regionais na distribuição da alergia tuberculínica foram registradas em outros paises. como nos $\mathrm{EI}^{-1}{ }^{21}, \cdots, \pm 9$. sendo atrihuidas solretudo à diversidade da situação sócio-econômica. a qual, por sua rez, condiciona maior ou menor incidencia da doença.

A explicação para a diversidade dos níveis da prevalência de infecção em $t$ cidades próximas deve estar relacionada à incidência da tuberculcse pulmonar no periodo de 10 anos. conforme demonstra a Tabela 4.

Wallgren. em $1956^{` 1}$, já havia levantado a possibilidade de se demonstrar essa relação numa coletividade. Bonilla, na Argentina ${ }^{\prime 2}$ e Neves no Espírito Santo ${ }^{28}$ também demonstraram essa associação.

Ao ser repetido o inquérito tuberculínico, na mesma população escolar de Conchas e São Manuel. em 1973. confirmouse o encontro de tavas ainda discrepan- te's. com maior concordância dos resultados de 1972 e 1973. em Conchas, conforme se observa na Tabela 5.

Quarenta e dois escolares de Conchas moravam em pequenos aglomerados fora do perímetro urhano e 148 na zona urhana. Entre os primeiros houve $9.52 \%$ de forte-reatores e entre os últimos $6,08 \%$.

Por outro lado. em São Manuel, entre 384 residentes na zona urbana, houve $1.56^{C} c$ de reatores-forles, enquanto que entre 73 da zona rural registrou-se $4,11 \%$.

Isto poderia indicar que, ao invés de menor risco. a residencia em zona rural, para as áreas em estudo. concorreu para elevar a probabilidade de infecção em crianças. Sabe-se que o sistema mais utilizado. atualmente, para arregimentar mão-de-otra para a zona rural é o que contrata trahalhadores diaristas ou "rolantes" os quais vivem com suas famílias em más condições de habitação. saneamento e alimentação na periferia dos centros urbanos. Esta situação deve ser uma das principais responsáveis pela chamada "interiorizaçãa" da tuberculose.

É provável que em Conchas as condições de transmissão do bacilo tuberculoso fossem ainda mais propícias. dada a existencia de hairros rurais e a verificação de cifras mais elevadas tanto de infecção em escolares como de incidência da doença. Investigações subseqüentes neste sentido são necessárias.

Em Botucatu e Araré. praticamente. todos os escolares examinados residiam em zona urhana.

Com referência ao risco de infecção. vê-se pela Tahela 6 que os indices de inridência anual. por idade. ohtidos pelos dois métodos, foram hastante similares ou iquais. O segundo método forneceu resultados pouco mais elevados: segundo Lotte e cols.". a fórmula estabelecida por Styblo e cols." evita que as taxas sejam subestimadas. isto é. para uma dada coorte sulimetida a um risco constante, o número de crianças infectadas annalmente tende a reduzir-se porquanto as que perma- 


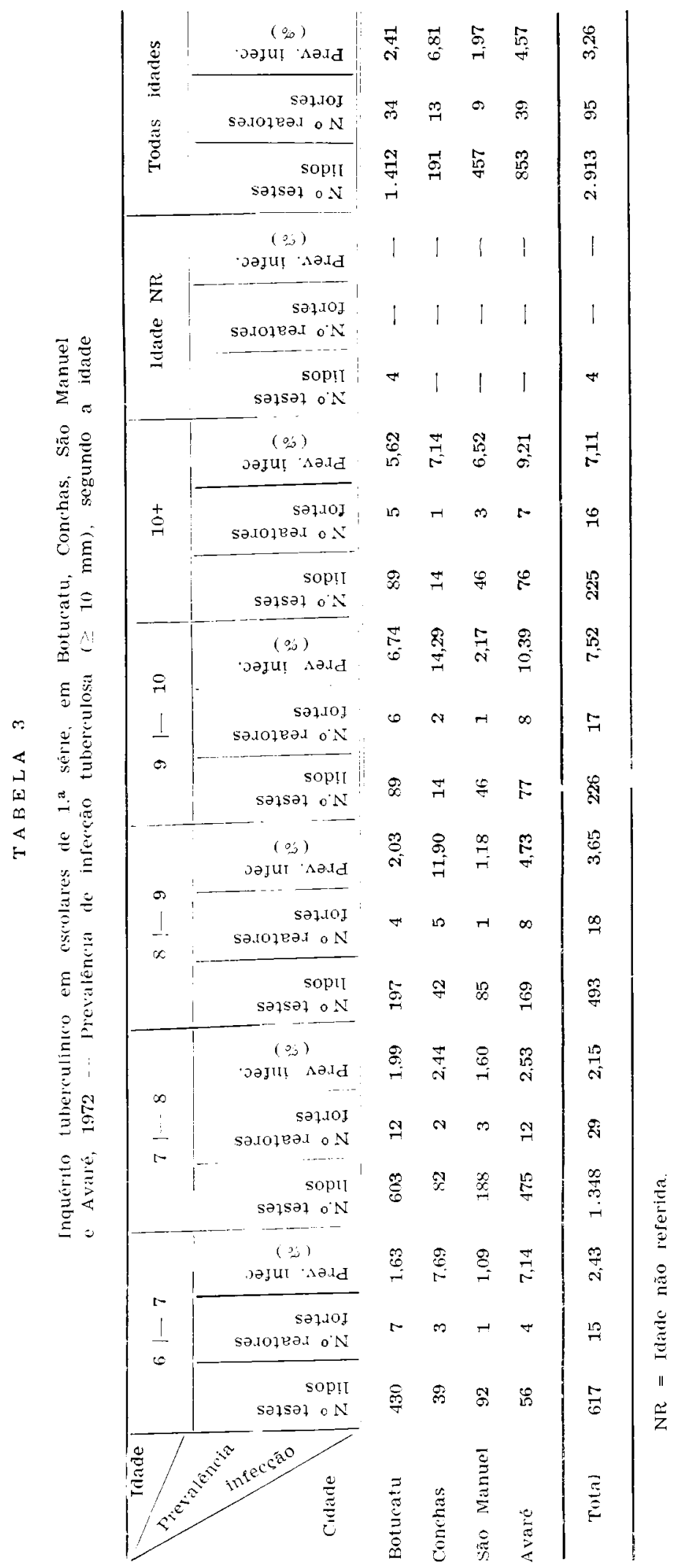


MAGALDI, C. et al. - Aspectos epidemiológicos da tuberculose pulmonar nos municipios de Botucatu, Conchas, São Manuel e Avaré, Estado de São Paulo, Brasil, de 1963 a 1972. Rev. Saúde públ., S. Paulo, 10:291-314, 1976.

\section{TABELA 4}

Indices de prevalência da infecção tuberculosa em escolares de 1.a série (1972) em Botucatu, Conchas, São Manuel e Avaré e indices de incidência da doença

\begin{tabular}{l|c|c|c|c}
\hline \multicolumn{1}{c|}{ Indices } & Botucatu & Conchas & São Manuel & Avaré \\
\hline $\begin{array}{l}\text { Prevalência de infec- } \\
\text { cão }(\%)-1972\end{array}$ & 2,41 & 6,81 & 1,97 & 4,57 \\
\hline $\begin{array}{l}\text { Incidência média da } \\
\text { doença (1963-1972) } \\
\text { por 100.000 hab. }\end{array}$ & 23,71 & 35,41 & 18,57 & 33,12 \\
\hline $\begin{array}{l}\text { Incidência da doença } \\
\text { (1972) por 100.000 } \\
\text { hab. }\end{array}$ & 26,04 & 49,21 & & 31,46 \\
\hline
\end{tabular}

T A B E L A 5

Prevalēncia de reatores-fortes à prova tuberculínica na mesma população escolar examinada em 1972 e 1973, Conchas e São Manuel

\begin{tabular}{|c|c|c|c|c|c|}
\hline \multirow{2}{*}{ Cidade } & \multirow{2}{*}{ Idade } & \multicolumn{2}{|c|}{ População examinada } & \multicolumn{2}{|c|}{ Prev. forte-reatores $(\%)$} \\
\hline & & 1972 & 1973 & 1972 & 1973 \\
\hline \multirow{6}{*}{ Conchas } & 6 & 39 & 一 & 7,69 & - \\
\hline & 7 & 82 & 39 & 2,44 & 7,69 \\
\hline & 8 & 42 & 49 & 11,90 & 4,08 \\
\hline & 9 & 14 & 33 & 14,29 & 15,15 \\
\hline & $10+$ & 14 & 14 & 7,14 & - \\
\hline & Total & 191 & 135 & 6,81 & 7,41 \\
\hline \multirow{6}{*}{ São Manuel } & 6 & 92 & 5 & 1,09 & 二 \\
\hline & 7 & 188 & 119 & 1,60 & 0,85 \\
\hline & 8 & 85 & 114 & 1,18 & 5,31 \\
\hline & 9 & 46 & 51 & 2,17 & 5,77 \\
\hline & $10+$ & 46 & 54 & 6,52 & 9,09 \\
\hline & Total & 457 & 343 & 1,97 & 4,37 \\
\hline
\end{tabular}

necem indenes no início de cada ano diminuem progressivamente.

O risco de infecção tuberculosa é tanto mais fidedigno como indicador da situação epidemiológica da tuberculose quanto mais baixos forem os grupos etários estudados para seu cálculo ${ }^{42}$. À idade de 6,5 anos, verifica-se que os riscos foram maiores em Conchas e Avaré $(1,20$ e $1,10 \%)$. Os índices de Botucatu e São 
MAGALDI, C. et al. - Aspectos epidemiológicos da tuberculose pulmonar nos municipios de Botucatu, Conchas, São Manuel e Avaré, Estado de São Paulo. Brasil, de 1963 a 1972. Rev. Saúde pübl., S. Paulo, 10:291-311. 1976.

\section{TA BELA 6}

Inquérito tuberculinico em escolares de 1 a sérle primária na zona urbana de Botucatu, Conchas, São Manuel e Avaré, 1972 - Estimativa de incidência de infecção tuberculosa, segundo a idade

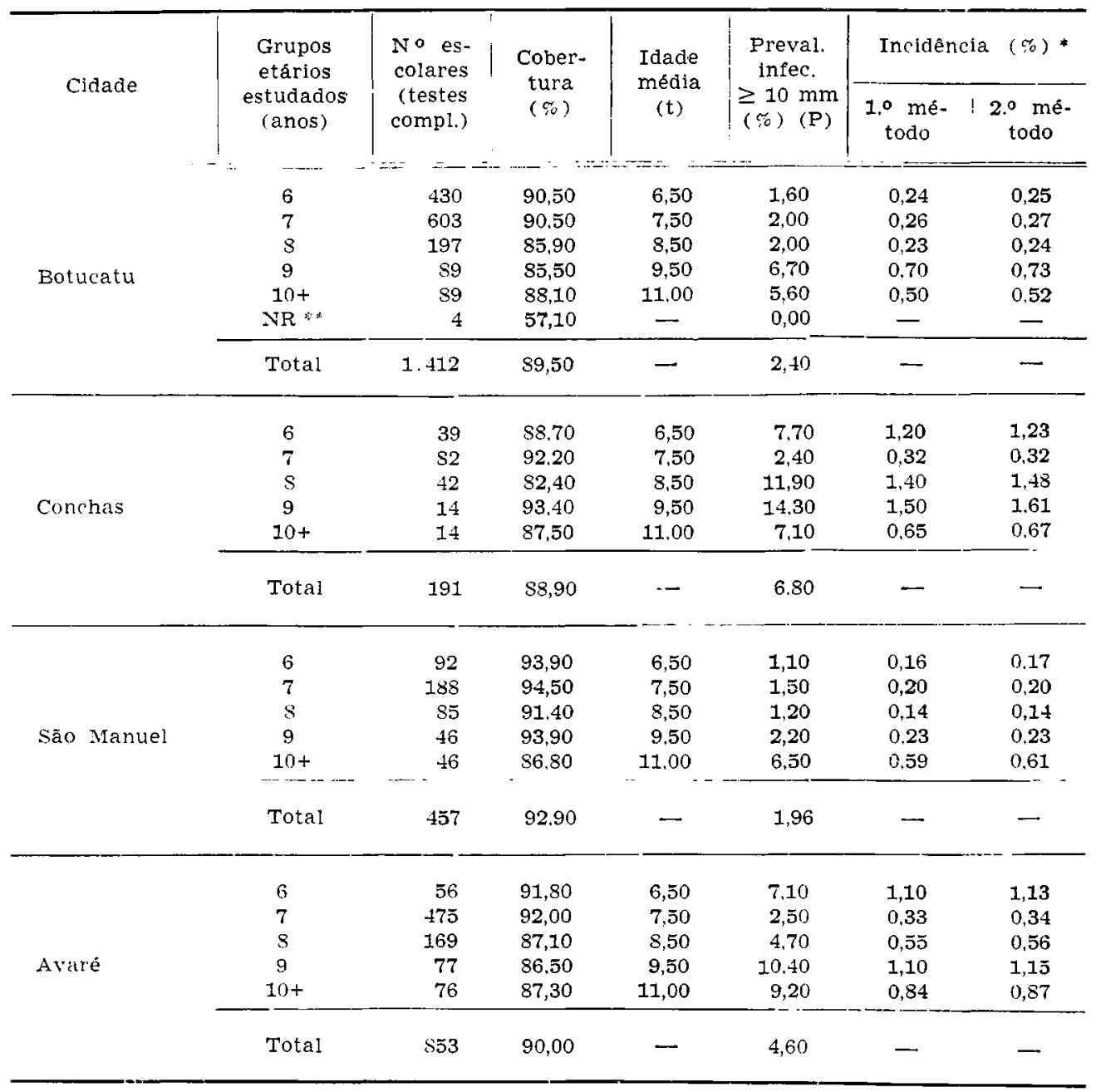

* 1.0 método: $i=\frac{P}{t} ; 2 .{ }^{\circ}$ método: Styblo et al is: $P(t)=1-(1-t)^{\dagger}$

* NR = Idade não referıda

Manuel foram semelhantes ao estimado $\mathrm{em}$ Petrolina (PE) por Lima ${ }^{31}$, em 82 escolares de 6.5 anos $(0.18 \%)$. e cerca de 12 vezes inferiores ao ohtido em Ilhéus (BA). em 84 crianças. pelo messmo autor". Admite-se que em Illhéus, cujo ris- ('o $(2.4 \%)$ foi duas vezes maior que o de Conchas. o elevado nível de infecção possa estar relacionado à alta incidência de casos novos que era registrada na zona cacaueira da Bahia até 1964 :?.

Os indices correspondentes à idade de 
MAGALDI, C. et al. - Aspectos epidemiológicos da tuberculose pulmonar nos municipios de Botucatu, Conchas, São Manuel e Avaré, Estađo de São Paulo, Brasil. de 1963 a 1972. Rev. Saúde públ., S. Paulo, 10:291-314, 1976.

7.5 anos para Conchas e Avaré são um pouco mais elevados do que os de Botucatu e São Manuel.

Sabe-se hoje que nos paises desenvolvidos o risco de infecção caiu abaixo de $0,5 \%$, enquanto em outro extremo, em áreas sub-desenvolvidas, o risco anual está acima de $2 \%{ }^{17}$. Nestes últimos, a tendência anual do risco vem se mantendo nos últimos 10 anos, enquanto nos primeiros, o decréscimo anual está ao redor de $10 \%$. Os índices para os quatro municípios em estudo estão em situação intermediária. A medida do risco anual e mais ainda a sua tendência histórica são de alto valor para estabelecer as diretrizes de programas de controle ${ }^{35}$.

\section{Morbidade}

Em 530 dos 718 casos inscritos $(73,8 \%)$ foi utilizada a baciloscopia para confirmação diagnóstica, com $62,08 \%$ de resultados positivos (329 casos).

A combinação de métodos diagnósticos mais utilizada foi a de exame clínico + $\mathrm{RX}+$ baciloscopia, com $65,18 \%$, principalmente no sexo masculino e idade adulta.

Autores nacionais têm apontado taxas de positividade à baciloscopia de 84 a $93 \%$, em outros serviços ${ }^{26,29}$, devendo-se salientar que, dentre 155 unidades da Fundação SESP com $93,1 \%$, somente 4 dispunham de aparelho de $\mathrm{RX}^{29}$. Diferenças de casuística, de técnicas de laboratório e/ou de critérios de caso novo podem ser a razão da menor taxa de positividade encontrada neste trabalho. Estes resultados serão úteis para comparação futura, após a intensificação do uso do diagnóstico bacteriológico como método de eleição para descoberta de $\operatorname{casos}^{14}$ $\$ 2,65$.

A distribuição dos casos por idade, sexo e formas segundo extensão das lesões radiológicas e critérios terapêuticos, fez-se de modo esperado, conforme demonstra a Tabela 7, ressalvando-se proporçóes ain$\mathrm{da}$ altas de forma mínima, moderada e avançada no grupo de 0-4 anos.

Chamou a atenção o fato de que, entre 170 pacientes de 20-29 anos, os percentuais de formas mínima, moderada e avançada foram respectivamente de $17,6 \%, 34,7 \%$ e $43,5 \%$, mostrando que na maioria das vezes, em que adultos jovens tiveram sua doença diagnosticada, ela já havia progredido bastante.

Quanto ao sexo, até 14 anos de idade, as formas primárias, forma mínima e derrame pleural tiveram a freqüência de $89,17 \%$ para o sexo masculino e $91,38 \%$ para o feminino. As formas moderada e avançada ocorreram em $10,83 \%$ dos homens e $8,62 \%$ das mulheres, taxas muito similares. Por outro lado, a partir de 15 anos de idade, as diferenças acentuaramse a favor do sexo feminino, com formas primária e mínima em $14,10 \%$ para o sexo masculino e $21,47 \%$ para o feminino; formas moderada e avançada em $83,61 \%$ para os homens e $77,40 \%$ para as mulheres. Para todas as idades, as formas moderada e avançada ocorreram em $63,06 \%$ dos homens e em $50,17 \%$ das mulheres. Pode-se supor que os homens adultos, além de submetidos a maiores tensões e desgastes físicos e conseqüente diminuição da resistência ${ }^{27}$, teriam menos motivação e facilidade para demanda aos serviços de saúde quando doentes.

Excluindo-se 152 casos de tuberculose primária "sem sombras" e 9 casos de forma não especificada, obteve-se um total de 557 casos cujas formas apresentavam as seguintes proporções:

$$
\begin{aligned}
& \text { Forma mínima } \ldots . .85(15,44 \%) \\
& \text { Forma moderada ... } 179(32,14 \%) \\
& \text { Forma avançada } \ldots 2.236(42,37 \%) \\
& \text { Derrame pleural ..... } 1(0,18 \%) \\
& \begin{array}{c}
\text { Forma primária com } \\
\text { sombras } \ldots \ldots \ldots \ldots
\end{array} 55(9.87 \%) \\
& \text { Total } \ldots \ldots .557(100,00 \%)
\end{aligned}
$$




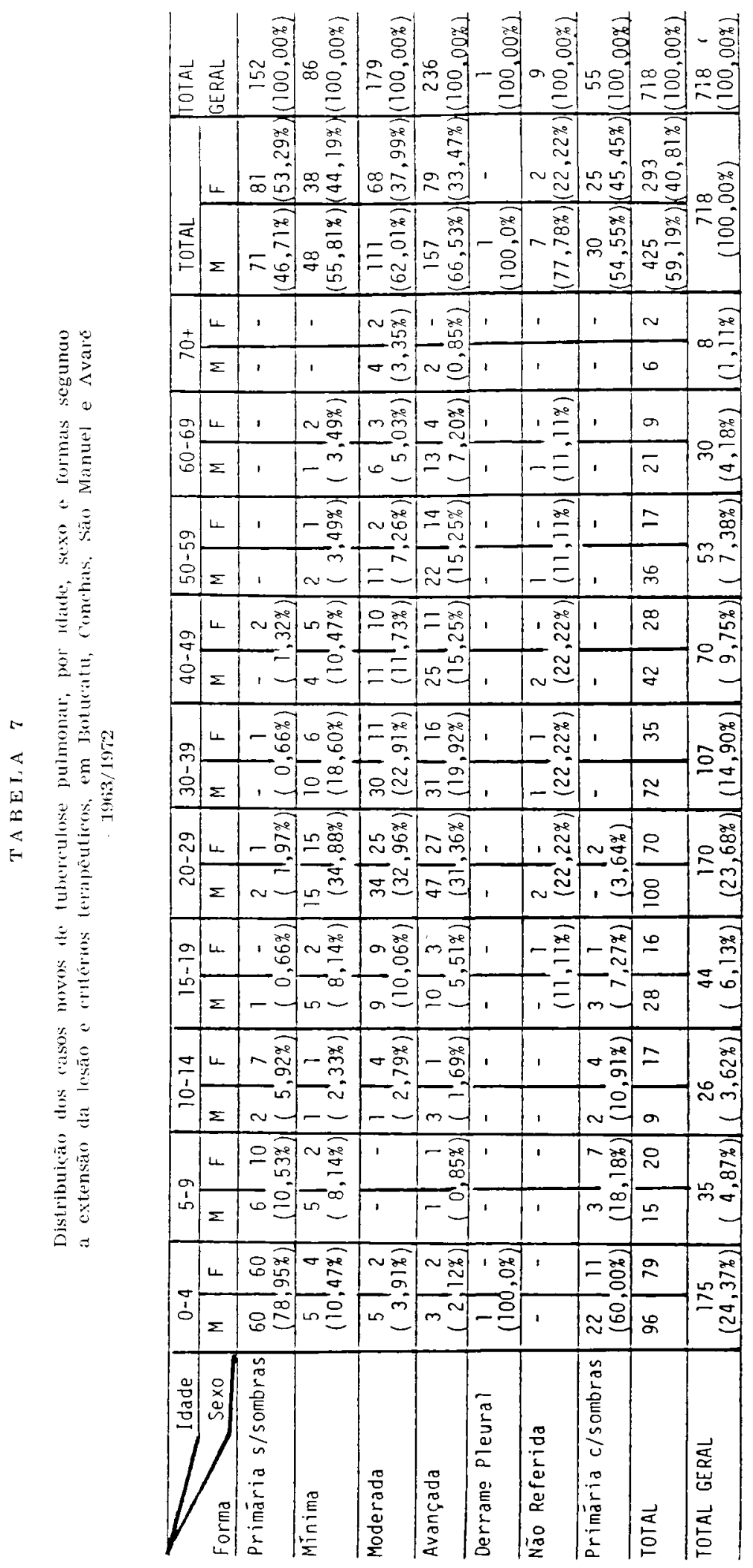


MAGALDI, C. et al. - Aspectos epidemiológicos da tuberculose pulmonar nos municípios de Botucatu, Conchas, São Manuel e Avaré, Estado de São Paulo, Brasil, de 1963 a 1972. Rev. Saúde públ., S. Paulo, 10:291-314, 1976.

Portanto. as formas moderada e avancada foram as mais freqüentes traduzindo diagnóstico tardio ou inscrição tardia no serviço. e. ainda. comprometendo mais indivíduos na fase produtiva da vida. Em outras áreas do País, altas proporções de formas mais arançadas de tuberculose também têm sido registradas $1, \cdots, 13,2 \pm, 26$, ". 6ib. sugerindo reduzidas atividades de proteção aos suscetíveis e infectados e de descoberta e tratamento precoce de doentes. por parte dos serriços.

Excluindo-se os 152 casos de tuberculose primária "sem sombras" e 55 de tuberculose primária "com sombras", $57.34 C_{C}$ dos pacientes foram tratados em regime monofásico e $+2.66 c_{c}^{c}$ em regime difásico.

Pelos dados da Tabela 8 calculou-se que, de 718 casos, $39.55 \% c$ abandonaram o tratamento. $17.97 \%$ curaram-se, $3,48 \%$ faleceram. 2,23c foram transferidos e $32.31 \% \mathrm{C}$ encontravam-se em tratamento. As informações foram desconhecidas em $4.3 \gamma_{c}$ dos casos. Verifica-se. também, pela análise de cada grupo etário. que em crianças e adultos jovens as percentagens de cura foram mais elevadas e as de óbito situaram-se por ordem decrescente nas faixas de 60 e mais anos. 50-59, 40-49, 15-19. 10-14 e 20-29. Nas áreas estudadas para o período. portanto, eram ainda elevadas as proporçốes de pacientes tuherculosos jovens que faleceram. Não houve disponibilidade de informaçōes sobre as causas de óbito. As proporções de abandono não guardaram estreita relação com a idade. Não houve diferenças importantes para os dois sexos quanto à evolução.

A comparação com resultados de tratamento obtidos em outros serviços, conforme mostra a Tabela 9, ressalvando-se as diferenças de critérios para "caso noro" e outros possíveis fatores, parece indicar que a eficiência do tratamento da tuberculose no Centro de Saúde de Botucatu não foi satisfatória. Os serviços de saúde de outros Estados conseguiram melhores resultados do que os divulgados para três unidades de saúde do Estado de São Paulo, em época anterior à reforma administratica da Secretaria da Saúde. As causas de insucessos foram muito bem analisadas por Rouillon ${ }^{5.1}$ : Teixeira ${ }^{69}$, Fox ${ }^{2-}$ e Neves ${ }^{39}$ e giram em torno da falta de uma "organização sistêmica. econômica e administratira suficiente e capaz". uma vez que a eficácia do tratamento com drogas de primeira linha pode alcançar de 85 a $95 \%$ de curas ${ }^{\$,}, 9 \pi, 4 \pi, 69$. A responsabilidade pelo fracasso terapêutico que se poderia atribuir a uma eventual resistência primária não subsiste diante dos achados de Mitchison citado por Teixeira ${ }^{6 !}$. segundo os quais. mesmo com um nivel de resistência de $30 \%$, o insucesso seria de $5.1 \%$. To presente trabatho apenas $18 \%$ dos casos curaram-se.

Analisando os prontuários de 232 pacientes classificados como "em tratamento", apurou-se que 73 casos estaram na realidade em abandono. sem o conhecimento formal do serviço, com o que a cifra global de abandono passou de $39.55 \%$ a quase $50 \%$. Este fato susciton o interesse en se rerificar se as taxas de abandono variavam com a residência do paciente e/ou com a forma da doença segundo a extensão das lesões, em 501 casos.

Os resultados das Tabelas 10 e 11 mostraram não haver associação sionificante entre abandono. procedência e forma da doença, tanto na análise conjunta como quando se considera cada uma das formas ou cada município isoladamente ou juntos. Nas áreas em estudo, portanto, contrariamente ao que foi observado em outros trabalhos ${ }^{37,45,54}$, tanto a distância como a gravidade da tuberculose não tiveram influência aparente no grau de abandono. devendo haver outras razôes para o fato. De modo geral, essas razóes constistiriam mais em fatores técnicos e administrativos do que comportamentais ${ }^{10}$. Com a descentralização das atividades anti-tuberculose e sua integração às demais 


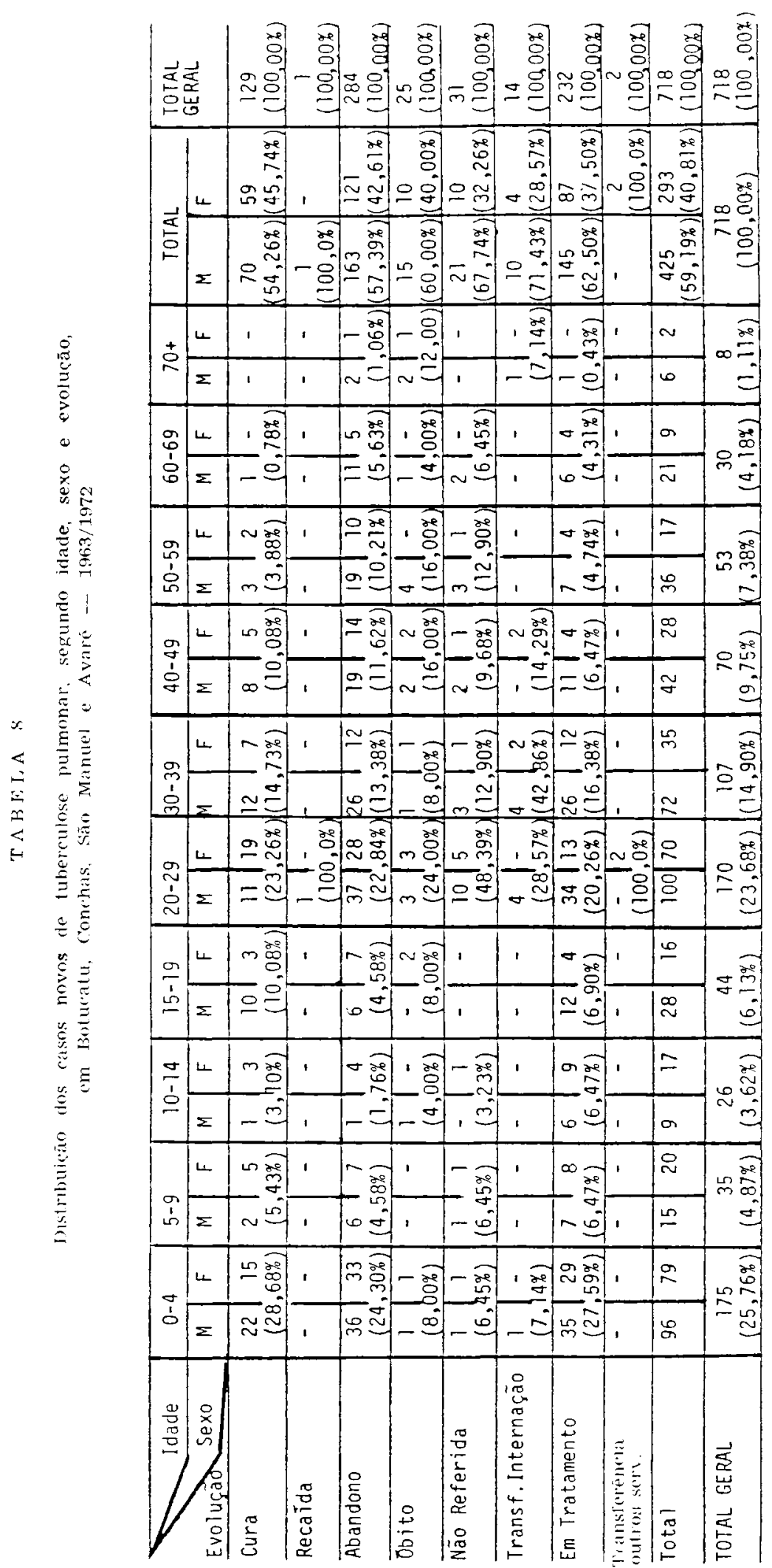


MAGALDI, C. et al. - Aspectos epidemiológicos da tuberculose pulmonar nos municipios de Botucatu, Conchas, São Manuel e Avaré, Estado de São Paulo, Brasll, de 1963 a 1972. Rev. Saúde puibl., S. Paulo, 10:291-314, 1976.

\section{TABELA 9}

Resultados comparativos quanto à cura, abandono e óbito, obtidos em diferentes serviços de saúde, no Brasil

\begin{tabular}{|c|c|c|c|c|c|c|}
\hline Autores e periodo & Local & $\begin{array}{l}\text { Tipos de } \\
\text { serviço }\end{array}$ & $\begin{array}{l}\text { Cura } \\
(\%)\end{array}$ & $\begin{array}{l}\text { Aban- } \\
\text { dono } \\
(\%)\end{array}$ & $\begin{array}{l}\text { obito } \\
(\%)\end{array}$ & $\begin{array}{l}\text { Total de } \\
\text { casos }\end{array}$ \\
\hline $\begin{array}{l}\text { Fraga et al. }{ }^{26} \\
1965-67\end{array}$ & Rio de Janeiro & $\begin{array}{l}\text { Disp.-Escola } \\
\text { (DNT) }\end{array}$ & 81,4 & 6,9 & 3,2 & 913 \\
\hline $\begin{array}{l}\text { Braga 1s } \\
1966\end{array}$ & $\begin{array}{l}\text { João Pessoa } \\
\text { (PB) }\end{array}$ & $\begin{array}{l}\text { Dispensário } \\
\text { (Secret. Saúde) }\end{array}$ & 70,2 & 23,6 & 3,0 & 145 \\
\hline $\begin{array}{l}\text { Fraga et al, }{ }^{28} \\
\text { 1966-67 }\end{array}$ & Brasil & $\begin{array}{l}17 \text { Disp. } \\
\text { (Serv. Est. } \\
\text { Saúde) }\end{array}$ & 65,3 & 14,0 & 3,5 & 3.349 \\
\hline $\begin{array}{l}\text { Castro Neto et } \\
\text { al. }{ }^{13} \\
1966-67\end{array}$ & Bahla & $\begin{array}{l}15 \text { Unidades da } \\
\text { Fundação SESP }\end{array}$ & 75,0 & 7,3 & 11,0 & 529 \\
\hline $\begin{array}{l}\text { Quilici \& Conde * } \\
\text { 1966-67 } \$\end{array}$ & Osasco (SP) & $\begin{array}{l}\text { Dispensário } \\
\text { (Secret. Saúde) }\end{array}$ & 42,5 & 20,0 & 1,6 & 120 \\
\hline $\begin{array}{l}\text { Arantes } \\
1969-71\end{array}$ & $\begin{array}{l}\text { Rib. Preto } \\
\text { (SP) }\end{array}$ & $\begin{array}{l}\text { Centro de } \\
\text { Saúde }\end{array}$ & 48,0 & 29,0 & 4,5 & 113 \\
\hline $\begin{array}{l}\operatorname{Lima}^{30} \\
1970\end{array}$ & Brasil & $\begin{array}{l}\text { Unidades da } \\
\text { Fundação SESP }\end{array}$ & 76,1 & 10,1 & 5,0 & 2.929 \\
\hline $\begin{array}{l}\text { Villas Boas } 79 \\
1971\end{array}$ & Brasil & $\begin{array}{l}\text { Com atividades } \\
\text { integradas }\end{array}$ & 73,3 & 11,2 & 5,3 & 3.290 \\
\hline $\begin{array}{l}\text { Magaldi } \\
1963-72\end{array}$ & $\begin{array}{l}\text { Botucatu, Con- } \\
\text { chas, São Ma- } \\
\text { nuel e Avare } \\
\text { (SP) }\end{array}$ & $\begin{array}{l}\text { Disp. ou Area } \\
\text { de Tisiologia } \\
\text { do CS-I de } \\
\text { Botucatu }\end{array}$ & 17,97 & 39,55 & 3,48 & 718 \\
\hline
\end{tabular}

- Considerados apenas doentes da forma moderada e avançada

TA B E L A 10

Distribuição dos doentes que abandonaram o tratamento, segundo municipios e formas de tuberculose

\begin{tabular}{|c|c|c|c|c|c|c|c|c|c|c|c|}
\hline \multirow[b]{2}{*}{ Forma } & \multicolumn{2}{|c|}{ Botucatu } & \multicolumn{3}{|c|}{ Conchas } & \multicolumn{2}{|c|}{ São Manuel } & \multicolumn{2}{|c|}{ Avaré } & \multicolumn{2}{|c|}{ Total } \\
\hline & $\begin{array}{l}\text { N.o } \\
\text { insc. }\end{array}$ & Aband. & $\begin{array}{l}\text { N.o } \\
\text { insc. }\end{array}$ & Abar & nd. & $\begin{array}{l}\text { N.o } \\
\text { insc. }\end{array}$ & Aband. & $\begin{array}{l}\text { N.o } \\
\text { insc. }\end{array}$ & Aband. & $\begin{array}{l}\text { N.o } \\
\text { insc. }\end{array}$ & Aband. \\
\hline Minima & 33 & 15 & 8 & 4 & 4 & 17 & 9 & 28 & 16 & 86 & 44 \\
\hline Moderada & 66 & 25 & 20 & 5 & 5 & 32 & 17 & 61 & 28 & 179 & 75 \\
\hline Avançada & 92 & 29 & 22 & 9 & 9 & 39 & 12 & 83 & 36 & 236 & 86 \\
\hline Total & 191 & 69 & 50 & 18 & & 88 & 38 & 172 & 80 & 501 & 205 \\
\hline Para forma minima & & & & $\chi^{2}$ & $=$ & 0,5214 & n.s. & g.1. & $=3$ & $=$ & 0,05 \\
\hline Para forma moderada & & & & $\chi^{2}$ & $=$ & 4,8424 & n.s. & g.l. & $=3$ & $\alpha$ & 0,05 \\
\hline Para forma avançada & & & & $\chi^{2}$ & $=$ & 3,4148 & n.s. & g.l. & 3 & $=$ & 0,05 \\
\hline
\end{tabular}


MAGALDI, C. et al. - Aspectos epidemiológicos da tuberculose pulmonar nos municípios de Botucatu, Conchas, São Manuel e Avaré, Estado de São Paulo, Brasil, de 1963 a 1972. Rev. Saúde públ., S. Paulo, 10:291-314, 1976.

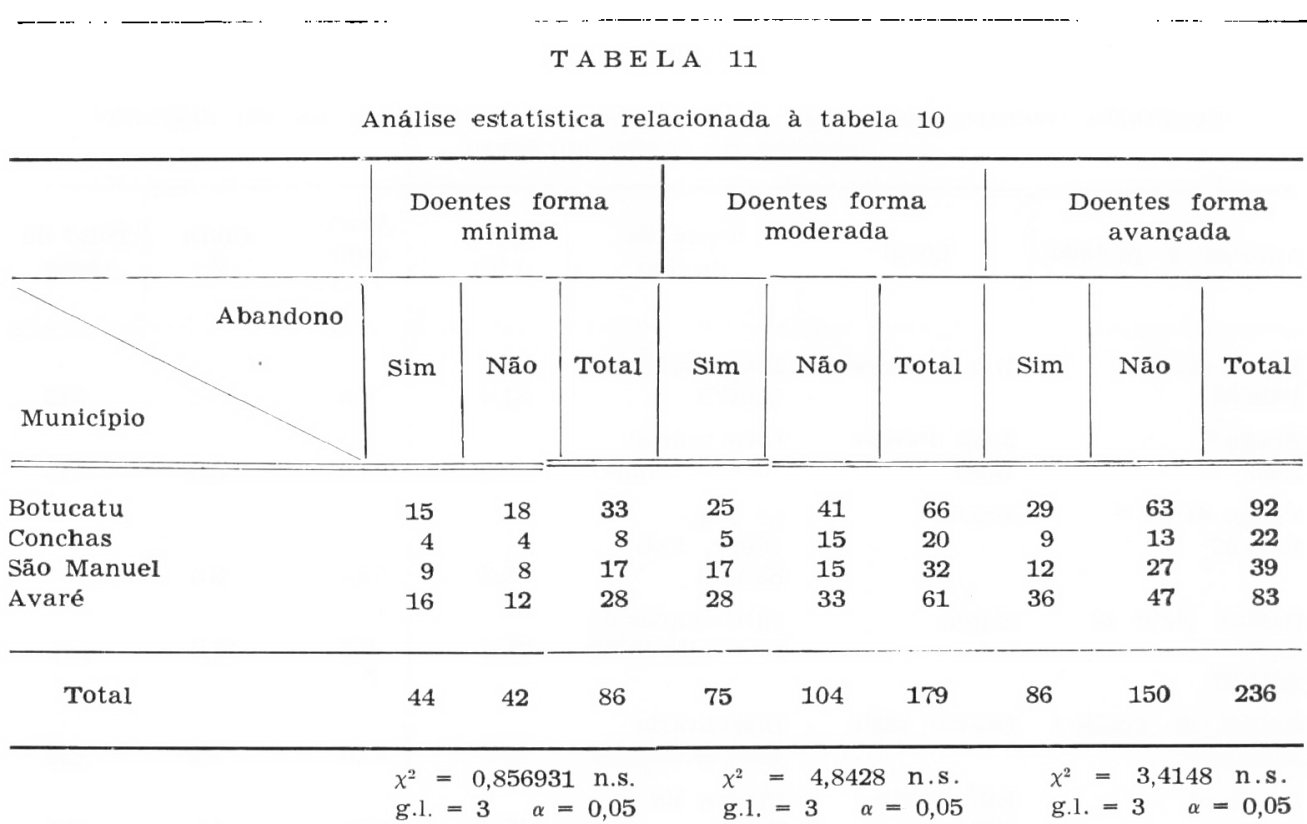

atividades nas unidades locais de saúde, metas prioritárias nos programas de controle da tuberculose $7,16,25,43,45,73$, ao lado de outros resultados favoráveis, espera-se uma redução significativa das taxas de abandono.

A incidência média anual (índice I) no período de 10 anos, tomando como numerador os casos com baciloscopia positiva diagnosticados no CSI de Botucatu, foi a seguinte: Conchas - 35,41; Avaré - 33,12; Botucatu - 23,71 e Sáo Manuel - 18,5, por 100.000 habitantes. $O$ indice de Conchas é quase duas vezes superior ao de São Manuel. Não foi evidenciada nos 4 municípios franca tendência ao declínio ou ascensão da incidência, nos 10 anos. Fatores biológicos e ambientais estariam interagindo no condicionamento da maior ou menor incidência da tuberculose ativa nos municípios estudados. Aspectos já mencionados na discussão dos níveis de infecção tuberculosa, provavelmente, incluem-se nesses fatores.

Quanto à incidência por idade, em 10 anos, o gráfico da Figura 1 mostra um padrão similar nos quatro Municípios, notando-se valores máximos no grupo de 20-29 anos.

Em paises desenvolvidos registrou-se deslocamento da maior incidência de casos para grupos de idade avançada ${ }^{33,67}$.

Calculando-se índices médios por idade para o primeiro e para o último triênio da série, observando-se o grupo etário de 20-29 anos, verificou-se que em Botucatu, São Manuel e Avaré os índices do primeiro triênio foram mais elevados. Em compensação, em Conchas, constatou-se fenômeno inverso, com um índice negativo de 1963 a 1965 e pouco mais de 120/ 100.000 nos anos de 1970 a 72 , o que constitui mais um elemento negativo com relação à tendência da tuberculose pulmonar nesse município. Sob o ponto de vista de incidência por idade e sexo, importa apenas assinalar que à idade de 20-29 anos, enquanto as diferenças entre os sexos em Botucatu e São Manuel foram insignificantes em Avaré e mais ainda em Conchas. o índice para o sexo masculino foi muito superior ao do sexo feminino, conduzindo à hipótese de excesso de risco 
MAGALDI, C. et al. - Aspectos epidemiológicos da tubereulose puimonar nos municípios de Botucatu, Conchas, São Manuel e Avaré, Estado de São Paulo, Brasıl, de 1963 a 1972. Rer. Salue publ, S. Paulo, 10:291-314, 1976.

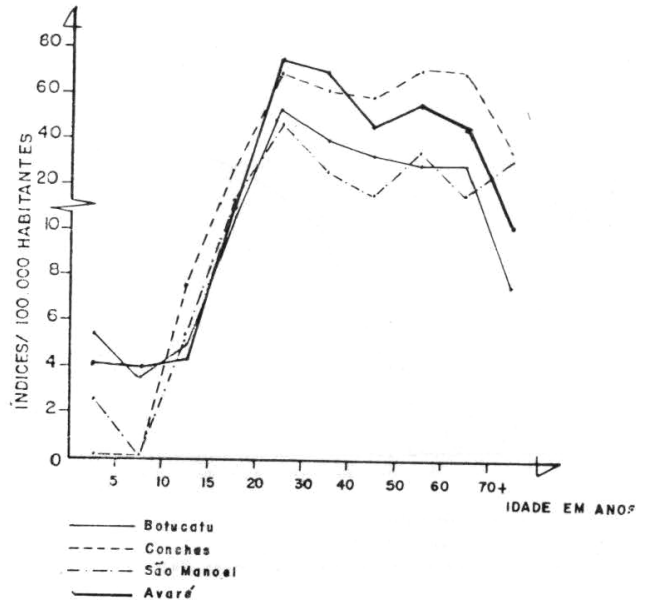

Fig. 1 - Incidencia média anual de tuberculose pulmonar por idade, nos municipios de Botucatu, Conchas, São Manuel e Avaré. (Casos novos com baciloscopia positiva) $1963-1972$.

e'm adultos jovens por fatores ainda desconhecidos.

Os índices de incidência de casos contfirmados são os mais fidedignos na definiçāo da morbidade por tuberculose pulmonar numa comunidade ${ }^{15,34,46,48}$, mesmo sabendo-se que nem todos os casos existentes são registrados ou conhecidos pelos serviços de saúde ${ }^{2}, 1 \overline{1}, 35.10 .70$.

Entretanto, a inobservância da conceituação uniforme de caso de tuberculose tem dificultado a comparação de dados oficiais de prevalência e/ou incidência entre países e áreas. Em geral. os dados existentes são de casos notificados com hase em confirmação ou mesmo na suspeita.

Os índices II. médio-anuais de casos confirmados e suspeitos foram os seguintes: Conchas - 57.66: Avaré - 48.88: Botucatu - 43.80 e São Manuel - 35.38 por 100.000 . Em confronto com índices de outros municípios do Brasil, baseados em casos confirmados ${ }^{\text {T2 }}$ e com o de casos notificados. estimado em 1973. para a Divisāo Regional de Saúde de Sorocaba ${ }^{60}$, estes valores mostraram-se inferiores. $O$ indice de Conchas foi 1.6 vezes maior que o de São Manuel.

Em suma. as informações de morbidade permitem admitir que: a) o risco de adoecer por tuberculose pulmonar diferiu em cada município; b) a distribuição das formas de tuberculose segundo extensão das lesões não apresentou nítidas diferenças entre os municípios. e c) os tipos de evolução também não mostraram tendências específicas por município.

Parece pois que. enquanto condicionados por fatores de cada estrutura epidemiológica. o evento incidência apresentou um quadro diversificado. Quando tiveram como denominador comum o sistema de atenção à saúde, os eventos como forma da doença e evolução mostraram um comportamento similar.

\section{Mortalidade especifica}

Para um total de 134 óbitos, em 10 anos. nos quatro municípios, a proporção de óbitos registrados fora dos municipios de origem (hospitais de Tisiologia) foi de $37.31 \%$. assim distribuída: Botucatu - 60.53\%: Conchas - 58.33\% ; São Manuel - 50.0\% e Avaré - 18.57\%

0 s indices médio-anuais na ordem acima. foram os seguintes: 7.63: 12,14; 5,00 e 18.70 por 100.000. sendo o de Avaré cerca de 3.5 vezes superior ao de São Vanuel.

O único declínio mais pronunciado e progressivo, na tendência da mortalidade nos 10 anos. verificou-se em Botucatu, com o indice de 1972 dez vezes inferior ao de 1963. Em centros urbanos maiores, no Brasil. foi assinalada expressiva queda na mortalidade até $1969^{1 \% 39,50}$.

A maior mortalidade por idade. como era esperado, evidenciou-se nos grupos acima de 50 anos. mas como mostra a Figura 2, os índices para os grupos de 20-29 e 30-39 foram elevados. demons- 
MAGALDI, C. et al. - Aspectos epidemiológicos da tuberculose pulmonar nos municipios de Botucatu, Conchas, São Manuel e Avaré, Estado de São Paulo, Brasil, de 1963 a 1972. Rev. Saúde plibl., S. Paulo, 10:291-314, 1976.

trando que adultos jovens ainda pagavam pesado tributo à tuberculose.

Foi estimado para os quatro municípios o indice médio por idade. para o primeiro e último triênio da série.

Além da redução do valor dos índices de um período para outro, como se verifica no gráfico da Figura 3 , notou-se que a taxa média no grupo de 20-29 anos reduziu-se de cerca de 5 vezes, enquanto no grupo de 60-69 o declínio foi de 2,5 vezes. o que demonstra uma tendência favorável na mortalidade em relação à idade. A redução observada deveu-se em grande parte à queda registrada em Botucatu. No mesmo gráfico, a curva relativa à mortalidade específica, por idade para o interior do Estado e corrigida por local de residência, evidencia um aumento progressivo a partir de 20 anos*.

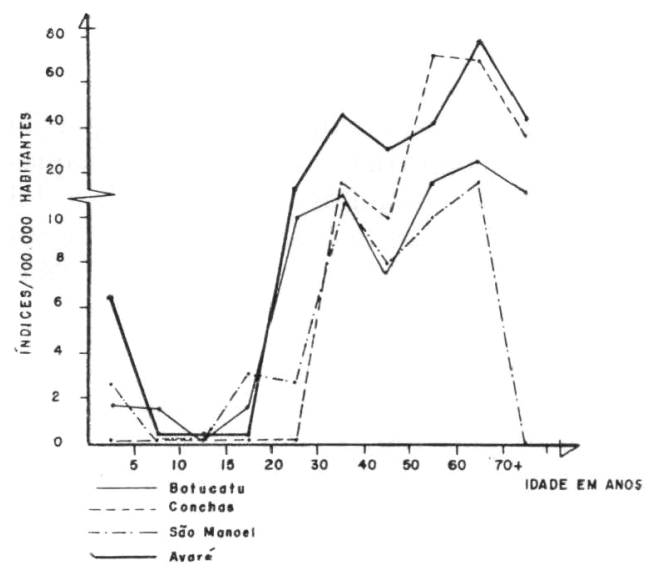

Fig. 2 - Mortalidade média anual por tuberculose pulmonar, segundo idade (1963 1972), nos municípios de Botucatu, Conchas, São Manuel e Avaré.

Quanto à mortalidade por idade e sexo, os índices masculinos foram sempre maiores, sendo dez vezes superior o risco

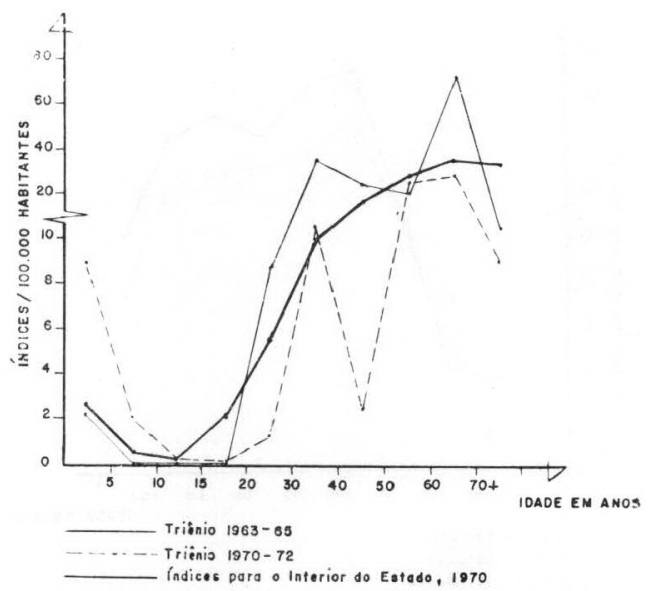

Fig. 3 - Mortalidade médla anual por tuberculose pulmonar segundo idade. Comparação de triênios extremos (indices para quatro municipios) e de indices para o interior do Estado.

de morrer por tuberculose pulmonar, em Conchas. para o homem em relação à muther.

\section{CONCLUSOES}

1. O nivel de infecção tuberculosa em escolares de primeira série da zona urba:ı de Botucatu, Conchas, São Manuel e Avaré. mensurado por inquérito tuberculínico, embora diferindo em cada cidade, caracterizou o conjunto estudado como área de média prevalência de tuherculose.

2. Os índices de prevalência de infecção relacionaram-se diretamente aos índices de incidencia de casos bacilíferos, nos municípios. ficando assim, em parte, explicadas as diferenças observadas na proporção de forte-reatores, em quatro cidades de uma mesma região.

- Dados procedentes, em números absolutos, do Departamento Estadual de Estatistica e fornecidos pelo Prof Dr. Edmundo Juarez. 
MAGALDI, C. et al. - Aspectos epidemiológicos da tuberculose pulmonar nos municiplos de Botucatu, Conchas, São Manuel e Avaré, Estado de São Paulo, Brasil, de 1963 a 1972. Rev. Saüde públ., S. Paulo, 10:291-314, 1976.

3. A tuberculose-doença, em formas avançadas, ainda comprometia em maior freqüência adultos jovens sobretudo do sexo masculino, nos municípios estudados.

4. A pequena proporção de cura e a elevada taxa de abandono, no período de dez anos, sugeriram baixa eficácia de tratamento e, em última análise, pouca eficiência das atividades de controle, pelo serviço.

5. A demonstração de que a distância entre a residência do paciente (tomando-se como referência as sedes municipais e o serviço especializado) e a forma de tuberculose não influenciaram o grau de abandono, levou à suposição de que deficiências técnico-administrativas do serviço foram os principais obstáculos para a regularidade do comparecimento dos doentes em tratamento.

6. A incidência de casos confirmados à baciloscopia, considerando-se todas as idades, manteve-se estável no período; todavia o deslocamento da incidência para as idades mais avançadas, no último triênio, indicou em Botucatu, São Manuel e Avaré, uma modificação favorável na tendência da doença.

7. Esboçou-se uma queda na mortalidade específica por tuberculose pulmonar, na medida em que se registrou um índice médio, no último triênio, cinco vezes inferior ao do primeiro para os adultos jovens, no conjunto dos quatro municípios.

8. Os dados indicativos da "interiorização" da tuberculose e os relativos ao insucesso da terapêutica, dentro da política de trabalho então vigente, apontaram a necessidade urgente de descentralizar as ações antituberculose, implantando-as em cada unidade de saúde da área, para um controle eficiente da tuberculose pulmonar. 
MAGALDI, C. et al - Aspectos epidemiológicos da tuberculose pulmonar nos municípios de Botucatu. Conchas. São Nanuel e Avaré, Estado de São Paulo. Brasil, de 1963 a 1972. Rev. Saúde puibl., S. Paulo, 10:291-314, 1976.

Magaldi, C. et al. - [Epidemiological aspects of pulmonary tuberculosis in Botucatu, Conchas, são Manuel and Avaré (State of S. Paulo), from 1963 to 1972]. Rev. Saúde públ., S. Paulo, 10:291-314, 1976.

SUmMary: The diagnosis of the pulmonary tuberculosis situation in Botucatu, Conchas, São Manuel and Avaré (State of S. Paulo) was based on the study of prevalence and risk of infection, in first grade school - children, during 1972, and on morbidity and specific mortality data gathered during a ten year period, 1963 to 19\%2. Tuberculin survey with PPD 23 RT-2TU was carried out in 2.913 school chitdren; 718 records from the Botucatu Public Health Center and the death certificates of residents who died in these connties or in specialized hospitals were analysed. The prevalence rates of infection for the 4 counties were respectuely 2.4.6.8.1.9 and $4.5 \%$. The risks of injection for 7.5 year old school-children were $0.27,032,0.20$ and $0.34 \%$. The infection level characterized these counties as constituting a medium prevalence area of pulmonary tuberculosis. The prevalence rates of infection were directly related to the incidence of smear positive cases, in all these counties. Sixty tuo percent (62.0) of 530 patients submitted to direct microscopy were smear positue cases. The predominance of moderately advanced and far advanced pulmonary lesions was observed in male young adults. A high default rate (39.5), a low proportion of cure (17.9\%) and death (3.4\%) were observed. Thirty two percent were under treatment. There were no statistically significant differences between default cases and county procedence of default cases and extent of pulmonary lesions. These findings were attributed to the present technical and administrative health organization. The average incidence of confirmed cases during this period was 35.4 in Conchas, 38.1 in Avaré, 23.7 in Botucatu and $18.5 \mathrm{in}$ São Manuel (per 100,000). The rates of confirmed and suspected cases together were: 5\%.6. 48.8,43.8 and 35.3. The average annual mortality rates were: 7.6 in Botucatu, 12.1 in Conchas, 5.0 in são Manuel and 18.7 in Avaré (m 100,000). The decreased mortality rates during 1963 to 1972 were observed only in Botucatu. Specific mortality is still high among the young male adults, specifically in Conchas. The need to incorporate the antituberculosis activities in local health services, for effective control of this disease was emphasized.

Uniterms: Tuberculosis putmonary. Tuberculin surey. school-chldren. Morbidity and mortality. Eplemiology.

\section{REFERENCIAS BIBLIOGRAFICAS}

1. ALBLQUERQUE, A. F. R. de \& VILLAS BOAS, A. - Aspectos epidemiológicos da tuberculose no Brasil. Rev, paut. Tisiol, 17:647-69, 1956.

2. ALBUQLERQUE. A. F. R. de \& VILLAS BOAS, A. - Revisão do valor dos indices epidemiológicos em tuberculose. Arq. Inst. bras. Invest. Tuberc., 19:160-5, 1960.

3. AlmeidA, A. P. de - Prevalência da infeção tuberculosa em escolares das capitais brasıleiras. Rev. Du. nac. Tuberc., 17:155-74, 1973.
4. ALMEIDA, A. P. de et al. - Levantamento da prevalência da infeçåa tuberculosa em escolares das capltais brasileiras: protocolo da investigaşão. Rev. Div. nac. Tuberc, 17: $176-98, \quad 1973$

5. ALMEIDA, E. S. de et al. - Infecção tuberculosa natural e o uso do BCG oral e intradérmico em escolares de Laranjal Paulista (SP), Brasil. Rev. Saúle púbt., S. Paulo, i:189-97, 1973.

6. Almeida, E. S. de et al, - Nivel de infeccão tuberculosa e viragem tu- 
MAGALDI, C. et al. - Aspectos epidemiológicos da tuberculose pulmonar nos municipios de Botucatu, Conchas, São Manuel e Avaré, Estado de São Paulo, Brasil, de 1963 a 1972. Rev. Saúde públ., S. Paulo, 10:291-314, 1976.

berculinica pelo BCG intradérmico em escolares de Botucatu (SP), Brasil, em 1969. Rev. Saúde públ., S. Paulo, 8:31-41, 1974 .

7. ARANTES, G. R. - Contribuição para a integração das atividades antituberculose nas unidades sanitárias do Estado de São Paulo. São Paulo, 1972. [Dissertação de Mestrado Faculdade de Saúde Pública, USP].

8. ARANTES, G. R. - Avaliaçāo de serviço anti-tuberculose na rotina de Saúde Pública. Rev. Saúde públ., S. Paulo, 8:105-18, 1974 .

9. ARANTES, G. R. - Planejamento anti-tuberculose. Valor predictivo do teste tuberculinico negativo; Influência da triagem tuberculinica no valor predictivo da abreugrafia. São Paulo, 1974. (Tese Doutoramento Faculdade de Saúde Pública, USP).

10. BANERJI, D. - Effect of treatment default on results of treatment in routine practice in India. Bull. Uni. int. Tuberc., 43:247-9, 1970.

11. BERQUÓ, E. - Bioestatística. São Pau10, Faculdade de Higiene e Saúde Pública, 1969. p. 166. [mimeografado]

12. BONILLA, J. L. - Programación de las actividades para el control de la tuberculosis. Torax, 14:4-14, 1965.

13. BRAGA, F. C. - Rendimento da quimioterapia standard, nos doentes V.T., no dispensário Cardoso Fontes (João Pessoa - PB): análise das dificuldades de sua aplicação e medidas susceptivels de melhorá-la. Rev. Serv. nac, Tuberc., 10: 463-6, 1966.

14. BRASIL. Ministério da Saúde - Ação antituberculose a nivel periférico. Brasilia, Ministério da Saúde/Ministério da Previdencia e Assistência Social, 1974. p. 120

15. CAMPANHA NACIONAL CONTRA A TUBERCULOSE. Comissão Técnica - Conceituação de "caso de tuberculose" na rotina em saúde pública. Rev. Serv. nac. Tuberc., 9:329-31, 1965.
16. CAMPANHA NACIONAI CONTRA TUBERCULOSE. Comissão Técnica - Prova tuberculinica em saúde Pública. 2. ${ }^{\text {a }}$ recomendação Rev. Serv. nac. Tuberc., 12:219-30, 1968.

17. CAMPOS, O. - Contribuicão para o planejamento da luta anti-tuberculose no Brasil. São Paulo, 1967. (Tese Livre-Docência - Faculdade de Higiene e Saúde Pública, USP).

18. CAMPOS, O. - A tuberculose no Brasil. Evolução e tendência. Rev. Serv. nac. Tuberc., 11:229-35, 1967

19. CASTRO NeTO, F. de P. et al. - Controle da tuberculose nas unidades da Fundação SESP, na Bahia. Rev. Serv. nac. Tuberc., 12:157-66, 1968.

20. DIXON, W. J. \& MASSEY JUNIOR, F. J. - Introduction to statistical analysis. $3^{\text {rd }}$ ed. New York, MacGrawHill, 1969 p. 237.

21. EDWARDS, L. B. \& PALMER, C. E. Tuberculosis infection. In: TUBERCULOSIS. Cambridge, Mass. Harvard University Press, 1969. p. 125-225.

22. EDWARDS, P. Q. \& EDWARDS, L. B. - Story of the tuberculin test from an epidemiologic viewpont. Am. Rev. resp. Dis., 81(suppl.):1-47, 1960.

23. FARO, M. de M. - Recenseamento torácico pelas unidades móveis de abreugrafia. Rev. Serv, nac. Tuberc., 10:69-75, 1966.

24. FERREIRA, J. C. \& JOUVAL JUNIOR, H. - Ação dispensarial antituberculose na prática de Saúde Pública. Rev. Serv. nac. Tuberc., 12:247-56, 1968.

25. FOX, w. - Organizational and administrative considerations in the diag. nosis and treatment of pulmonary tuberculosis in the developing countries. Tubercle, 49:332-46, 1968.

26. FRAGA, H. et al. - Controle do tratamento de tuberculose pulmonar em áreas urbanas do Brasil. Rev. Serv. nac. Tuberc., 13:329-49, 1969.

27. KAYNE, G. G. et al. - Pulmonary tuberculosis, Pathology, diagnosis, ma- 
MAGALDI, c. et al. - Aspectos epidemiológicos da tuberculose pulmonar nos municípios de Botucatu, Conchas, São Manuel e Avaré, Estado de São Paulo, Brasil, de 1963 a 1972. Rev. Saúde públ., S. Paulo, 10:291-314, 1976.

nagment \& prevention. London, Oxford University Press, 1948. p. 651.

28. LIMA, L. L. de - Atividades de luta contra a tuberculose integrada nos programas gerais de saúde pública. Rev. Serv. nac. Tuberc, 13:33-48, 1969 .

29. LIMA, L. L. de - A expansão da luta contra a tuberculose no Brasil. Rev. Div. nac. Tuberc., 14:279-82, 1970.

30. LIMA, L. L. de - Controle da tuberculose nas unidades de saúde da Fundação SESP. Rev. Fund. SESP, $17: 61-8,1972$

31. LIMA, L. L. de - Implantação do BCG intradérmico nas unidades da Fundação SESP. Rev. Fund. SESP, 11: 46-79, 1972 .

32. LOTTE, A. et al. - Epidémiologie de la tuberculose et défaillances de la lutte antituberculeuse chez l'enfant. Bull. Org. mond. Santé, 44(supl.): 5-229, 1971.

33. LOWELL, A. M. - I Tuberculosis: morbidity and mortality and its control. In: TUBERCULOSIS. Cambridge, Mass., Harvard University Press, 1969. p. 1-121.

34. MAHLER, H. - Elementos básicos para el conocimiento del problema de la tuberculosis en una comunidad: epidemiologia, sociodemografia, recursos. Washington, D.C., Organización Panamericana de la Salud, 1965. p. 80-104. (OPS. Publ, cient., 112)

35. MARTINEZ, R. A. - Información estadistica necesaria para la formulación de un programa antituberculose y para la evaluación de la ejecución del programa. Venezuela, 1974. 17 p. [Apresentado ao 7.0 Congreso Venezuelano de Tisiologia e Neumologia, Caracas, 1974 e $18 .^{\circ}$ Congreso Panamericano de Tuberculosis y Enfermedades del aparato respiratório]

36. MENDONCA, L. T. de - Epidemlologia da tuberculose em escolares. J. bras. Med., 26:73-84, 1974.

37. NAGPAUL, D. R. et al. - A socio-epldemiological study of outpatients attending a city tuberculosis clinic in India to judge the place of specialized centers in a tuberculosis control programe Bull. Wld Flth Org., 43:17-43, 1970.

38. NEVES, J. S. - Elementos básicos para o conhecimento da extensão do problema da tuberculose: indices de infecção tuberculosa com a "prova tuberculínica standard" (PPD-Rt 23) particularmente nos individuos abaixo de 15 anos de idade. Rev. Serv. nac. Tuberc., 12:13-25, 1968.

39. NEVES, J. S. - Programa para o controle da tuberculose. Diagnóstico da situação, centralização, integração e avaliação. Rev. Div. nac. Tuberc., 16:421-39, 1972 .

40. NEVES, J. S. et al. - Revisão do valor dos indices epidemiológicos em tuberculose, Arq. Inst. bras. Invest. Tuberc,, 19:166-86, 1960.

41. NUNES, R. J. de L. - Prevalência de tuberculose - infeccão e doenca numa área do Municipio de Osasco. São Paulo, 1970. 40 p. (Dissertacão de Mestrado - Faculdade de Saúde Pública, USP).

42. NYBOE, J. \& CIIRISTENSEN, O. W. Measurement of the incidence of tuberculosis infection. Bull. Wld Flth Org., 35:547-55, 1966.

43. OLIVEIRA, A. G. - Plano de expansão dos servicos de tuberculose do Es. tado de São Paulo. Programa integrado de controle da tuberculose. João Pessoa, 1972. 24 p. (Apresentado ao $16.0^{\circ}$ Congresso Nacional de Tuberculose, João Pessoa, 1972).

44. EVALUACION de las recomendaciones formuladas por un grupo de expertos de la OMS en tuberculosis. Cronica de la OMS, 28:487-94, 1974.

45. ORGANIZACION MUNDIAL DE LA SALUD. Bureau Regional de l'Europe. L'intégration de la lutte antituberculeuse dans l'activité des servides de santé genéraux. Copenhagen, 1973. p. 31. (EURO, 1202).

46. ORGANIZACION MUNDIAL DE LA SALUD. Comite de expertos en Tuberculosis, Ginebra, 1964. Octavo in. 
MAGALDI, C. et al. - Aspectos epidemiológicos da tuberculose pulmonar nos municípios de Botucatu, Conchas, São Manuel e Avaré, Estado de São Paulo, Brasil, de 1963 a 1972. Rev. Saúde publ., S. Paulo, 10:291-314, 1976.

forme. Ginebra, 1964. (Ser. Inf. tecn., 290)

47. ORGANIZACIÓN MUNDIAL DE IA SALUD. Tuberculosis in the world. $\mathrm{Tb} / W P 73,7: 1-57,1973$.

48. ORGANIZACION MUNDIAL DE LA SALUD. Regional Office for Europe. The effectiveness of tuberculosis control programmes. Copenhagen, 1972. 25 p. (EURO, 1201).

49. PALMER, C. E. et al. - Studies of pulmonary findings and antigen sens1tivity among student nurses. VI Geographic differences in sensitivity to tuberculin as evidence of nonspectfic allergy. Publ. Hith Rep., 65: $1111-28,1950$

50. PASsos FIlHo, M. C. da R. - Epidemiologia da tuberculose. Anexo 2. Relatório apresentado ao Grupo de Trabalho. In: SÃO PAULO (Estado). Secretaria de Saúde. Projeto para a realização de ensaios controlado de cotejo entre as técnicas oral e parenteral de vacinação pelo $B C G$. São Paulo, 1971. p. 13 [mimeografado].

51. QUILICI, B. \& CONDE, M. - Ação dispensarial antituberculose na prática de saúde püblica. Rev. Serv. nac. Tuberc., 13:351-60, 1969.

52. REGIONAL SEMINAR ON TUBERCULOSIS, 2.\%, Bogotá, 1972. Washington, D.C. Pan American Health Association, 1973. p. 5. (Sclent. publ., 265)

53. ROSEMBERG, J. et al. - o dispensário na profílaxia da tuberculose. I. Formas clínicas e resultados de tratamento dos doentes matriculados espontaneamente no Instituto Clemente Ferreira. Rev. bras. Tuberc., 16: 267-93, 1947.

54. ROUILLON, A. - Problemes posés par l'organisation d'un traitement ambulatoire efficace des malades tuberculeux: motivation. Bull. Un, int. Tuberc., 47:72-87, 1972.

55. RUFFINO NETO, A. - Epidemiologia $d a$ tuberculose. Estudo de alguns aspeotos ligados a modelos de preven. cão, diagnóstico e modelos epidemio. métricos. Ribeirão Preto, 1975. 94 p.
[Tese Livre-Docência - Faculdade de Medicina de Ribeirão Preto, USPJ.

56. SAO PAULO (Estado). Secretaria da Saúde. Reforma administrativa: textos legais e regulamentares 1967. 1970. São Paulo, Setor de Gráflca Dpto. Psiquiátrico, 1971.

57. SAO PAULO (Estado), Secretaria da Saúde. Ato do secretário. Normas para internação. Diário Oficial, São Paulo, 21 set. 1964. p. 38, Tc.

58. SAO PAULO (Estado), Secretaria da Saúde. A to do Secretário. Transferência do Municipio de Itatinga para o Distrito Sanitário de Botucatu. Diário Oficial, São Paulo, 10 mar. 1971. p. 40 .

59. SAO PAULO (Estado). Secretaria da Saúde, Conselho Técnico Administrativo. Norma técnica SS-5/70. Qulmioterapia da tuberculose pulmonar. Principios básicos e normas técnicas para sua aplicação. Diário oficial, São Paulo, 5 jan. 1971. p. 28-32.

60. SAO PAULO (Estado). Secretaria da Saúde. Departamento Técnico Normativo. Divisão de Epidemiologia. Análise dos coeficientes de morbidade por doenças transmissiveis segundo divisões regionais de saúde. Bol. Epidem. anual, 3:1-14, 1974.

61. SAO PAULO (Estado). Secretaria da Saúde. Divisão do Serviço de Tuberculose. Terapêtica da tuberculose pulmonar. Diârio Oficial, Sāo Paúlo, 28 lev. 1961. p. 31.

62. SAO PAULO (Estado). Secretaria da Saúde. Divisão do Servíço de Tuberculose. Normas técnicas do Setor de tratamento das unidades da divisão do serviço de tuberculose. São Pau. 1o, 1966. [mimeografado]

63. SAO PAULO (Estado). Secretaria da Saúde. Divisão do Serviço de Tuberculose. Normas técnicas de tratamento, São Paulo, 1968. [Orđem de serviço n.o 4/68 - mimeografado]

64. SAO PAULO (Estado). Secretaria da Saúde, Divisão do Serviço de Tuberculose. Normas técnicas de trata. 
MAGALDI, C. et al. - Aspectos epidemiológicos da tuberculose pulmonar nos municipios de Botucatu, Conchas, São Manuel e Avaré, Estado de São Paulo, Brasil, de 1963 a 1972. Rev. Saúde públ., S. Paulo, 10:291-314, 1976.

mento. São Paulo, 1968. [Ordem de serviço n.o $3 / 67$ - mimeograLado]

65. SENTIES, R. V. - Nuevos enfoques para el control de la tuberculosis. $S a$ lud pübl. Mex., 13:603-9, 1971.

66. SILVA, G. R. da - Morbidade por tuberculose pulmonar. Anexo 3 . Relatório apresentado ao grupo de trabalho. In: SAO PAULO (Estado). Secretaria da Saúde. Projeto para a realização de ensaio controlado de cotejo entre as técnicas oral e parenteral de vacinacão pelo $B C G$. São Paulo, 1971. p. 5. [mimeogra. 1ado]

67. STYBLO, $\mathrm{K}$. et al. - Epidemiological and clinical study of tuberculosis in the district of Kolin, Czechoslovakia: report for the first 4 years of the study (1961-64). Bull. Wld Hlth org., 37:819-74, 1967 .

68. STYBLO, $\mathrm{K}$. et al. - La transmission du bacille tuberculeux. Son evolution aux sein d'une collectivité humaine. Bull. Org. mond. Sante, 41: 137-78, 1969.
69. TEIXEIRA, G. M. - Quimioterapla de la tuberculosis. Progresos recientes aplicables a los programas de salud publica, Bol. Ofic. sanit. panamer. $74: 425-40,1973$.

70. TIZES, R. et al. - The source of notification in tuberculosis. Amer. J. publ. Hlth, 64:809-11, 1974.

71. U.S. NATIONAL TUBERCULOSIS AND RESPIRATORY DISEASE. Diagnostic standards classification of tuberculosis, New York, 1969. p. 75.

72. VILIAS BOAS, A. - A tuberculose no interior do Brasil. Rev. Serv. nac. Tuberc., 10:161-77, 1966.

73. VILLAS BOAS, A. - O controle da tuberculose na rotina de serviços gerais de saúde. Rev. Fund. SESP, 17: 9-36, 1972 .

74. WALLGREN, A. - La détermination de l'incidence de la tuberculose au moyen des tests tuberculiniques. Bull. Un. int. Tuberc, 26:707-9, 1956.

Recebido para publicação em 28/05/1976 Aprovado para publicacão em 14/06/1976 